\title{
Programa de tratamento assertivo na comunidade (PACT) e gerenciamento de casos (case management): revisão de 20 anos da literatura
}

\author{
Program for Assertive Community Treatment (PACT) and \\ Case Management: review of 20 years of literature \\ Louise de França Machado', Catarina Magalhães Dahl', Maria Cecília de Araújo Carvalho², \\ Maria Tavares Cavalcanti ${ }^{\top}$
}

\section{RESUMO}

Objetivo: Realizar uma revisão de estudos sobre programa de tratamento assertivo na comunidade (PACT) e case management para verificar se os resultados demonstram desfechos mais favoráveis quando tais modelos são implementados na rede comunitária de assistência para portadores de doença mental grave e persistente. Métodos: A coleta de artigos - publicados entre 1985 e 2005 - foi realizada em duas etapas: a primeira, na base de dados PubMed, com expressões-chave mental health, community care, services evaluation e seleção de artigos cuja temática era PACT e case management, e a segunda, no banco de dados da revista Psychiatric Services, com palavras-chave assertive community treatment, PACT e case management. Foram desconsiderados estudos que analisavam serviços exclusivos para crianças, idosos e pacientes com diagnóstico único de abuso de álcool/drogas; abordavam unicamente os custos da intervenção e se referiam exclusivamente a serviços hospitalares. Resultados: A partir da leitura dos 73 estudos selecionados, os autores des-

\section{Palavras-chave}

Case management, desinstitucionalização, serviços comunitários em saúde mental.

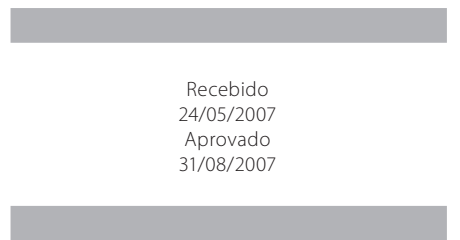

Recebido
24/05/2007

Aprovado

$31 / 08 / 2007$
1 Instituto de Psiquiatria da Universidade do Brasil/Universidade Federal do Rio de Janeiro (IPUB/UFRJ).

2 Escola Politécnica de Saúde Joaquim Venâncio (EPSJV/Fiocruz).

Endereço para correspondência: Louise de França Machado

Rua Campos Sales, 116, ap. 301/bloco 01 - Tijuca - 20270-270 - Rio de Janeiro, RJ

Telefone: $9923-0685$

E-mail: louise_machado@yahoo.com.br 


\section{Key-words}

Case management, community mental health services, deinstitutionalization. the articles with PACT and Case Management as subjects and the second one, from the journal Psychiatric Services database, with key-words assertive community treatment, PACT and case management. Articles that analyzed exclusive services for children, aged and patients with substance abuse as single diagnosis; approached only the costs of the intervention and related exclusively hospital-based services were not considered. Results: From the reading of the 73 selected articles, the authors had described 8 categories in which the articles had been gathered. One article could belong to more than one category. Conclusions: The PACT and case management are important and admittedly more efficient strategies, when compared with other models of care, in bringing favorable evolutions for individuals with serious and persistent mental illness.

\section{INTRODUÇÃO}

A reforma psiquiátrica é uma questão recorrente quando se discute a assistência, especialmente em relação às conseqüências da desinstitucionalização e reinserção de pacientes longamente institucionalizados na família e na comunidade.

A princípio, a desinstitucionalização buscava evitar internações sem indicação adequada, promover a volta à comunidade dos pacientes cuja hospitalização já não tivesse mais caráter terapêutico e estabelecer uma rede comunitária de suporte e assistência contínua (Amarante, 2000).

No entanto, os serviços comunitários que deveriam constituir alternativa ao modelo asilar acabaram, em parte, reproduzindo a cronificação e o isolamento. Segundo Mowbray et al. (1997-a), o que comumente se observou nos Estados Unidos, nos anos 1970, foi a desospitalização desacompanhada de suportes comunitários adequados. Eram freqüentes as queixas de que fora transferido para os familiares o "peso" de se cuidar de indivíduos cronicamente doentes, estigmatizados, excluídos e ignorados pela rede pública de saúde (idem).

As reinternações eram freqüentes, originando o fenômeno de porta giratória ou revolving door (Bandeira et al., 1998) e a desinstitucionalização foi acusada de ter provocado desassistência, já que "as condições que levaram o doente ao asilo, sejam clínicas, sejam econômicas, ou mais freqüentemente ambas, continuam a agir" (Cavalcanti, 1992, p. 142).

Neste contexto, surgem, na década de 1970, dois modelos de cuidado comunitário contínuo e intensivo: Case Management (Gerenciamento de Casos) e PACT (Program for Assertive Community Treatment ou Programa de Tratamento Assertivo na Comunidade).

\section{Case management}

No case management, um profissional é responsável pelo gerenciamento dos cuidados de determinado paciente na comunidade. Tal agente (case manager) deve promover o acesso aos serviços (psiquiátricos, clínicos, sociais) por ações de coordenação e integração da rede de saúde comunitária
(Bandeira et al., 1998). O conjunto das principais práticas terapêuticas do case management e os seus graus de importância encontram-se na tabela 1.

Tabela 1. Graus de importância das práticas terapêuticas, segundo especialistas em PACT e em case management

\begin{tabular}{|c|c|c|}
\hline Práticas terapêuticas & PACT & $\begin{array}{l}\text { Case } \\
\text { management }\end{array}$ \\
\hline Política de "não-alta" ("no-close" policy) & ++++ & +++ \\
\hline Seguimento assertivo e persistente & ++++ & ++++ \\
\hline Admissão de novos clientes em ritmo controlado & ++++ & +++ \\
\hline Critérios de admissão específicos & ++++ & +++ \\
\hline $\begin{array}{l}\text { Abordagem in vivo (nos locais de } \\
\text { moradia/trabalho/lazer do paciente) }\end{array}$ & ++++ & +++ \\
\hline $\begin{array}{l}\text { Baixa taxa cliente-terapeuta } \\
\text { (cerca de 10:1) }\end{array}$ & +++ & +++ \\
\hline $\begin{array}{l}\text { Oferecimento de suporte para obtenção de serviços } \\
\text { nos centros comunitários de saúde }\end{array}$ & +++ & ++++ \\
\hline Trabalho a partir do potencial do cliente & ++++ & ++++ \\
\hline Consulta dos clientes antes de decisões importantes & +++ & ++++ \\
\hline $\begin{array}{l}\text { Reuniões de equipe freqüentes } \\
\text { (número de reuniões/semana) }\end{array}$ & ++++ & ++ \\
\hline Disponibilidade da equipe por 24 horas & ++++ & ++++ \\
\hline Trabalho em equipe & +++ & +++ \\
\hline Compartilhamento de casos & ++++ & +++ \\
\hline Apoio na procura de trabalho & ++++ & +++ \\
\hline
\end{tabular}

As abordagens terapêuticas do case management agrupam-se em duas modalidades. Uma delas é o case management de agenciamento (brokering case management), no qual a equipe é responsável pela inserção do paciente no meio extrahospitalar por meio de ações de abordagem e monitoração da evolução do cliente, articulações entre os serviços da rede e representação de seus interesses na comunidade. A outra é o case management clínico (clinical case management), no qual os profissionais possuem maior qualificação e colocam-se como terapeutas primários, pois, além das ações de agenciamento, realizam intervenções clínicas terapêuticas (Bachrach, 1992). 
No entanto, na prática, tal divisão não é tão nítida, uma vez que os programas tendem a ficar entre os pólos, adotando tanto práticas de manejo clínico quanto de agenciamento. Ademais, verifica-se uma tendência ao deslocamento cada vez maior do case management para o perfil clínico (idem).

\section{Programa de tratamento assertivo na comunidade (PACT)}

O PACT surge nos EUA a partir da idéia de que a assistência predominantemente hospitalar não estava mais sendo efetiva para pacientes com funcionamento sociofamiliar precário, limitações nas atividades da vida diária e na resolução de problemas, alto grau de dependência e quadro clínico grave e instável (Dixon, 2000a). A solução deveria ser, então, mudar o foco de um modelo hospitalocêntrico para uma abordagem voltada para os recursos comunitários (idem), uma vez que "a comunidade tende mais a cobrar um comportamento adequado, a possuir modelos de funcionamento saudável e a permitir que o treinamento de habilidades seja especificamente orientado para as dificuldades do dia-a-dia" (Thompson et al., 1990, p. 626).

Assim, foi criado um programa cujo objetivo era fornecer suporte intensivo e orientado pelas necessidades do paciente (Tibbo et al., 1999), favorecendo a retomada de uma vida normal na comunidade e evitando internações. Nascia o community treatment group (grupo de tratamento comunitário) e, para demonstrar sua eficácia, foi feito um estudo controlado randomizado comparando-o com a assistência no contexto hospitalar (Dixon, 2000a). Observou-se, além da diminuição das reinternações, que "os pacientes do grupo de tratamento na comunidade não foram simplesmente transferidos para instituições na comunidade; eles viviam e trabalhavam em situações de autonomia. Eles também não foram simplesmente trazidos de volta às suas famílias. Os sintomas não pioraram" (idem, p. 760).

Diante destes resultados positivos, o Instituto Nacional de Saúde Mental passou a financiar, no Instituto de Saúde Mental de Mendota, em Madison, o training in community living program (programa de treinamento para a vida em COmunidade) que, posteriormente, passou a chamar-se PACT (Dixon, 2000a). O conjunto das principais práticas terapêuticas deste modelo aparece na tabela 1.

Tendo em vista a importância da pesquisa voltada para a identificação, conceituação e avaliação de dispositivos de cuidado comunitário em saúde mental, seriam o PACT e o case management produtores de desfechos clínicos e sociais mais favoráveis que outros serviços de saúde mental comunitários ou hospitalares?

O objetivo deste trabalho é realizar uma revisão de estudos sobre o PACT e o case management, a partir da coleta de artigos em base de dados virtual, selecionando aqueles que conceituam, descrevem e discutem tais modelos e suas adaptações, traçam a história dos programas, os comparam a outros dispositivos de assistência (serviços comunitários incluindo os centros comunitários de saúde mental/CMHC - e hospitalares), comparam o PACT com o case management, avaliam desfechos e eficácia, acessam o grau de satisfação com os programas, descrevem e analisam suas características e práticas e delineiam o estado da pesquisa sobre o tema. A revisão tem como objetivo conceituar o PACT e o case management e descrever suas características, funcionamento e adaptações, bem como verificar se os resultados dos estudos apontam para a obtenção de evoluções mais favoráveis para os pacientes portadores de doença mental grave e persistente quando comparados a outros serviços em saúde mental, o PACT e o case management são aplicados como estratégia de assistência comunitária.

\section{MÉTODOS}

Trata-se de uma revisão bibliográfica de estudos sobre o PACT e o case management.

A primeira coleta dos artigos foi feita na base de dados PubMed, a partir das expressões-chave mental health, community care, services evaluation e teve como limites da busca o idioma (inglês), a idade (adultos) e o período de publicação (1985-2005). Foram desconsiderados artigos que analisavam serviços de atendimento exclusivo para usuários de álcool/ drogas e idosos, discutiam predominantemente os custos da intervenção e se referiam exclusivamente a hospitais e serviços hospitalares. A acessibilidade dos periódicos e artigos por revista também foi considerada critério de busca.

Em seguida, foram separados para esta revisão os artigos cuja temática era o PACT e o case management.

Por ter sido a primeira coleta bastante ampla, contemplando vários outros tipos de serviços e aspectos da assistência comunitária que não faziam parte desta revisão, foi realizada uma segunda busca, mais específica, focalizando sobre os termos-chave assertive community treatment, PACT e case management e utilizando a base de dados da revista Psychiatric Services, uma vez que a maior proporção (46\%) dos artigos separados na primeira coleta era de publicações desta revista. Esta coleta teve como limites o período de publicação (1985-2005) e o idioma (inglês). A seleção de artigos obedeceu aos mesmos critérios de exclusão da primeira coleta.

\section{RESULTADOS}

Foram encontrados 73 estudos dos tipos controlado randomizado, de coorte, caso-controle, quase-experimental, avaliação experimental pós-teste, metanálise, revisão sistemática, revisão bibliográfica, qualitativo, descritivo/conceituação, relato de caso e apanhado histórico. 
Os estudos foram agrupados em oito categorias desenvolvidas a partir da análise dos objetivos de cada artigo.

A primeira categoria refere-se ao estudo de definição elou discussão do conceito de PACT ou case management. Nesta categoria, os artigos têm como objetivo fornecer definições gerais e princípios da assistência, delinear a população-alvo e discutir o papel das equipes terapêuticas (Bachrach, 1992; Bachrach, 1993; Bandeira et al., 1998; Drake; Burns, 1995; Kanter, 1989; Phillips et al., 2001; Witheridge, 1989). Tais artigos, de maneira geral, não se referem a programas específicos, mas Bachrach (1989) foca sobre dois programas de case management para exemplificar os conceitos de case management de agenciamento e de case management clínico (Commonwealth of Pensylvania e Community Connections, respectivamente) e discutir sobre tal multiplicidade de definições.

Phillips et al. (2001) descreveram os princípios e discutiram a implementação do modelo PACT com o objetivo de familiarizar e orientar os profissionais de saúde mental. Segundo os autores, o PACT direciona-se para pacientes com doença mental crônica e grave que possuem história de internações repetidas, má qualidade de vida e dificuldade em se manterem estáveis na comunidade. Os autores descrevem os princípios do PACT: oferecer ações de apoio, reabilitação e tratamento; praticar a atenção assertiva, mantendo a continuidade do tratamento; assumir total responsabilidade pelos clientes; ter baixa taxa cliente-terapeuta (cerca de 10:1); oferecer ampla gama de serviços/cuidados de modo flexível e individualizar as estratégias terapêuticas segundo as necessidades do paciente; atuar próximo aos pacientes; estar disponível 24 horas por dia; e oferecer assistência contínua, sem limite de duração.

Bachrach (1993), em uma análise crítica dos conceitos das duas modalidades de case management, aponta que, enquanto as equipes da modalidade de agenciamento geralmente possuem pouco treinamento em habilidades clínicas, os profissionais da modalidade clínica são altamente qualificados e oferecem diretamente cuidados terapêuticos. Por isso, a escolha da modalidade deve ser compatível com o tipo de assistência a ser oferecida, considerando quais as necessidades de cada população-alvo se deseja contemplar. A autora aponta que a escolha do profissional deve relacionar o grau e o tipo de formação à função a ser exercida. Além disso, recomenda-se que, mais do que apenas escolher entre as modalidades, os esforços terapêuticos devam ser direcionados a oferecer abordagens variadas (combinando as características das modalidades), de acordo com as necessidades dos clientes.

Na segunda categoria, estão os artigos referentes à história dos programas. O objetivo é traçar um histórico dos modelos desde sua criação até os dias atuais, podendo falar sobre disseminações do programa, mudanças em suas práticas ao longo do tempo e impacto sobre a assistência comunitária (Dixon, 2000a; Thompson et al., 1990).

Dixon (2000a) descreve a história do PACT desde suas ações "embrionárias", o community treatment group (grupo de tratamento comunitário), até o PACT atual, e constrói um panorama da assistência psiquiátrica da época, visando salientar as justificativas e os fatores que propiciaram a criação do programa; define o modelo PACT; fala sobre avaliações de eficácia e discute a evolução do programa ao longo dos anos, abordando assuntos como adaptações e disseminações e fidelidade ao programa. Aponta, também, algumas críticas baseadas na revisão de estudos sobre o PACT e o impacto desta iniciativa sobre a assistência para pacientes com doença mental grave e persistente.

A terceira categoria é o estudo de desfecho, cujos artigos possuem objetivo de verificar se a participação no PACT ou no case management provocou mudanças em um ou mais desfechos determinados pelos pesquisadores. Geralmente, são feitas análises e comparações entre as situações pré e pós-implementação dos programas em um mesmo serviço ou entre a situação no momento de entrada no serviço e a evolução depois de um período determinado pelo pesquisador (Borland et al., 1989; Curtis et al., 1996; Dixon et al., 1993a; Dixon et al.,1993-b; Joannette et al., 2005; Lang et al., 1999; McGrew et al., 1995; Shaw et al., 1990; Tibbo et al., 1999; Wasylenki et al., 1993).

Wasylenki et al. (1993) mediram o impacto da participação no case management sobre uma população de desabrigados com doença mental comparando as situações anterior e posterior à entrada no programa. Foram observadas melhoras significativas da estabilidade habitacional, do funcionamento social e de alterações psicopatológicas e aumento da rede social.

Dixon et al. (1993b) estudaram o nível de adesão ao tratamento global e a aspectos específicos do projeto terapêutico em pacientes desabrigados inseridos no PACT. Observou-se que mais da metade do grupo estudado foi capaz de aderir ao tratamento global e à maioria dos aspectos específicos.

McGrew et al. (1995) avaliaram desfechos de seis PACT implementados simultaneamente e observaram, após a entrada no programa, diminuição progressiva da freqüência e duração das internações. A partir da avaliação feita pelos técnicos da equipe e dos próprios pacientes sobre seu rendimento, viu-se que os indicadores de qualidade de vida melhoraram e que os cuidadores apontaram mudanças positivas no funcionamento/suporte familiar, nas habilidades da vida diária e no grau de autonomia/autoconfiança dos pacientes. No entanto, o desfecho "status laborativo" não mostrou melhora significativa, especialmente no tocante à inserção em empregos competitivos. Os pesquisadores consideraram este último achado como esperado, visto que não havia atividades de orientação vocacional. 
Tibbo et al. (1999), em um estudo de coorte, observaram o número e a duração de internações de pacientes com doença mental grave inscritos no PACT. Foram coletados e comparados dados de um ano antes e após a entrada no programa. Observou-se que, um ano após a entrada, houve diminuição no número e na duração da hospitalização por paciente e na freqüência em serviços de emergência psiquiátrica.

A quarta categoria é o estudo de comparação entre PACT/ case management e outros serviços (comunitários ou hospitalares) de saúde mental, no qual os pesquisadores também analisam desfechos para comparar o PACT/case management com outros serviços (Bond et al., 1988; Dincin et al., 1993; Franklin et al., 1987; Goering et al., 1998; Herinckx et al., 1997; Lafave et al., 1996; McRae et al., 1990; Morse et al., 1992). Pode haver, também, comparações entre o case management oferecido isoladamente e o associado a outras abordagens terapêuticas (Clark; Rich, 2003; Macias et al., 1994) e entre o PACT e o case management (Essock; Kontos, 1995; Schaedle et al., 2002 - tabela 1).

Clark e Rich (2003), em um estudo prospectivo quaseexperimental, comparam a efetividade do case management oferecido isoladamente com uma associação case management - programa habitacional. Os pacientes foram divididos em grupos segundo o grau de disfuncionalidade. Observou-se que aqueles com alto grau de disfuncionalidade obtiveram maiores ganhos habitacionais com a associação case management - programa habitacional, enquanto os pacientes com disfuncionalidade leve e moderada obtiveram ganhos com o case management isoladamente. Tais achados sugerem que "as taxas de efetividade podem ser aumentadas pelo pareamento cuidadoso entre as intervenções e as características dos usuários" (Clark e Rich, 2003, p. 82).

Lafave et al. (1996) compararam os desfechos de uma amostra de pacientes alocada randomicamente no PACT e em programas de reabilitação com base hospitalar. Os pacientes do PACT tiveram menor duração de internação, meIhor situação habitacional e maiores escores de satisfação com o tratamento e sensação de autonomia.

Com o objetivo de fazer uma comparação entre o PACT e o case management, Essock e Kontos (1995), em um estudo controlado randomizado realizado em serviços de alto padrão de qualidade, analisaram medidas de desfecho de ambos os programas. Observou-se que os grupos inseridos no PACT obtiveram menores duração de internação e instabilidade no padrão habitacional. Este estudo descreve o processo de implementação de equipes PACT, motivo pelo qual ele foi inserido também na sexta categoria.

A quinta categoria é a descrição e/ou estudo de desfecho de PACT ou case management que adotam abordagens fora do conjunto das práticas tradicionais dos programas. Este item diz respeito às adaptações (mudanças nas características originais ou adição de novas práticas) dos programas
(Becker et al., 1999; Dixon et al., 1999; Liberman; Kopelowicz, 2002; Meisler et al., 1997; Neale; Rosenheck, 2000; Rosenheck; Neale, 2004). Os artigos têm como objetivo descrever tais adaptações, podendo fazer estudos de desfecho. Nesta categoria, estão as descrições de PACT e case management para populações-alvo específicas, como população rural, pacientes violentos, minorias étnicas e clientes com comorbidade de uso de álcool/drogas (Durell et al., 1993; Dvoskin; Steadman, 1994; Fariello; Scheidt, 1989; Santos et al., 1993; Yang et al., 2005). Outra adaptação bastante estudada é a inclusão, na equipe terapêutica, de indivíduos idosos ou portadores de doença mental (Dixon et al., 1997; Nikkel et al., 1992; Sherman; Porter, 1991; Simon et al., 1993).

Meisler et al. (1997) descreveram um PACT adaptado voltado para garantir a continuidade do cuidado no período entre a alta hospitalar e a inserção em acompanhamento regular na rede de serviços comunitários. A diferença entre tal adaptação e o PACT tradicional estava na duração e no objetivo do tratamento: enquanto o PACT visa a reabilitação e integração na comunidade e preconiza o cuidado por tempo prolongado (Schaedle et al., 2002), esta adaptação oferecia tratamento de curta duração (por constituir uma transição entre o hospital e os serviços comunitários) e voltava-se para a estabilização dos sintomas psiquiátricos e satisfação de necessidades materiais/sociais do cliente. Os autores avaliaram os efeitos da adaptação após um ano de funcionamento e concluíram que tal iniciativa mostrou-se capaz de garantir a manutenção da continuidade do cuidado no pós-alta hospitalar.

A sexta categoria é o estudo/descrição das características e práticas dos programas PACT e case management. Os artigos têm como objetivos, a partir da coleta de dados sobre determinadas iniciativas de case management ou PACT, sistematizar as características, práticas e/ou funcionamento dos programas, descrever o processo de implementação dos modelos e/ou analisar cada característica separadamente. Divide-se em cinco subcategorias:

\section{Características/funcionamento}

Descrição das características e/ou funcionamento do programa, incluindo avaliações de eficácia e estudo tipo relato de caso (Kanter; Silva, 1996). Este subitem focaliza em programas de PACT e case management específicos e, geralmente, já estabelecidos (Deci et al., 1995; Mowbray et al.,1997-a; Ridgely et al., 1996).

Ridgely et al. (1996) sistematizaram as funções das equipes de case management clínico e de agenciamento dos programas da Fundação Robert Wood Johnson para Doença Mental Crônica. São elas: acompanhamento psiquiátrico com manejo da medicação, psicoterapia/aconselhamento, suporte habitacional e laborativo, realização de atividades sociais, abordagem do uso de álcool/drogas, suporte em atividades diárias e manejo do dinheiro, agenciamento de 
cuidados clínicos/odontológicos, busca ativa, treinamento em habilidades sociais e em autocuidado e defesa dos interesses do cliente.

Mowbray et al. (1997a) descreveram as características e o processo de avaliação da primeira replicação com alta fidelidade do modelo PACT: o programa de harbinger of grand rapids. O programa foi avaliado, a partir de estudo de casocontrole, em dois tempos após sua implementação. No primeiro momento, foram vistos melhores resultados no grupo experimental nos desfechos funcionamento, situação laborativa, hospitalização e moradia. Já no segundo momento, houve menos diferenças entre os grupos. Os autores concluíram que o PACT é um método bem-sucedido de assistência comunitária e que pesquisas sobre o tema devem continuar sendo feitas, especialmente em relação à detecção dos elementos preditivos de melhores desfechos.

\section{Avaliação dos desfechos/eficácias}

Avaliação se determinadas características do programa (por exemplo, fidelidade aos princípios do PACT) pode influenciar nos desfechos e/ou na eficácia global do modelo (Bush et al., 1990; Curtis et al., 1992; Dietzen e Bond, 1993; Dixon et al., 2000-b; Hornstra et al., 1993; Huxley e Warner, 1992; Joannette et al., 2005; King et al., 2000; McHugo et al., 1999; Neale e Rosenheck, 1995). Também estão incluídos os artigos referentes à identificação dos elementos críticos do PACT e do case management sem os quais a eficácia e o funcionamento ficariam comprometidos (Fiander e Burns, 2000; McGrew et al., 2003) e à delimitação, a partir destes elementos, das diferenças entre o PACT e o case management (Schaedle et al., 2002 - tabela 1).

Schaedle et al. (2002) entrevistaram experts em PACT e em case management para identificar os principais elementos de cada programa e evidenciar características que possam diferenciar o PACT do case management (Tabela 2). Os autores também observaram que os dois programas possuem muitos elementos importantes em comum, no entanto, o PACT apresenta-se como o modelo mais claramente articulado.

Outro ponto estudado é a fidelidade dos programas aos princípios tradicionais do PACT, em que se procura saber quão próximo da situação ideal estão trabalhando as replicações/adaptações dos modelos. McHugo et al. (1999), em um estudo quase-experimental, examinaram a relação entre o grau de fidelidade aos princípios do PACT e determinados desfechos nas populações de programas para pacientes com diagnóstico duplo. Os programas foram classificados como de alta e baixa fidelidade, de acordo com o grau de observância aos princípios do PACT listados pelos pesquisadores. Observou-se que não houve diferenças entre os grupos de alta e baixa fidelidade na duração das internações, nos sintomas psiquiátricos, nas relações familiares/sociais e na satisfação com a própria vida e com o serviço. No entanto, no grupo de alta fidelidade, houve grandes redução e remissão do uso de álcool/drogas, melhores taxas de adesão ao tratamento e menor número de internações psiquiátricas.

McGrew et al. (2003) identificaram, a partir da visão de equipes de PACT, elementos críticos do programa. Após a análise dos dados, viu-se que os elementos foram colocados na seguinte ordem decrescente de importância para o funcionamento ideal: enfermeira em tempo integral; acompanhamento nas internações e na alta; plano terapêutico elaborado pela equipe; menos de 100 pacientes inscritos; reuniões diárias; proporção cliente-terapeuta menor que 12:1; maioria dos serviços oferecidos em casa/comunidade; casos compartilhados; equipe como terapeuta primária; assistente social em tempo integral; psiquiatra presente mais

Tabela 2. Comparação das características do PACT e dos programas dos centros comunitários de saúde mental (CMHC)

\begin{tabular}{|c|c|c|}
\hline Característica & PACT & CMHC \\
\hline Base do tratamento & Predominantemente na comunidade & Na comunidade, mas predominantemente na clínica \\
\hline Equipe & Taxa terapeuta:cliente cerca de 1:10 & Taxa terapeuta: cliente cerca de 1:30 a 1:50 \\
\hline Freqüência do contato & Diariamente, na maioria dos casos & Usualmente a cada uma ou duas semanas \\
\hline $\begin{array}{l}\text { Freqüência do contato com família } \\
\text { ou estrutura de suporte }\end{array}$ & Média de uma vez/semana & Ocasional \\
\hline Medicação & $\begin{array}{l}\text { Responsabilidade da equipe; pode ser administrado diariamente } \\
\text { pela equipe, caso necessário }\end{array}$ & Responsabilidade do paciente ou da família \\
\hline Saúde física & Monitorada pela equipe do programa & 0 terapeuta e o case manager encorajam um estilo de vida saudável \\
\hline Serviço após o expediente & Monitorado pela equipe do programa; equipe disponível & $\begin{array}{l}\text { Oferecido pelo terapeuta ou case manager durante o dia, } \\
\text { emergência ou equipe móvel em outros casos }\end{array}$ \\
\hline Reabilitação ocupacional & Inserção em trabalho ou em serviços voluntários & Programas psicossociais \\
\hline Questões habitacionais & Responsabilidade da equipe & Variável, mas usualmente é responsabilidade do cliente e da família \\
\hline Continuidade do cuidado & $\begin{array}{l}\text { A equipe acompanha o caso durante a internação; mantém sistema } \\
\text { legal e de saúde, e outros contatos }\end{array}$ & Responsabilidade do terapeuta e do case manager \\
\hline Estrutura da equipe & $\begin{array}{l}\text { Estrutura de equipe: integração dos papéis clínico e } \\
\text { de agenciamento do caso }\end{array}$ & $\begin{array}{l}\text { Modelo de terapeuta individual: o terapeuta e } 0 \\
\text { case manager são indivíduos diferentes }\end{array}$ \\
\hline
\end{tabular}

Tradução de tabela extraída de Taube et al.. 1990, p. 643. 
do que oito horas/semana; critérios de admissão objetivos; abordagem assertiva; oferecimento de sistema de suporte; contatos semanais com o paciente; poucas trocas dos membros da equipe; oferecimento direto de serviços; líder da equipe clínica é membro da equipe; acompanhamento contínuo; líder da equipe realiza diretamente os serviços; responsabilidade total pelo cliente; reuniões de equipe que duram menos que uma hora; especialistas em abuso de substâncias, orientação vocacional e em moradia em tempo integral e nunca dispensar pacientes.

\section{Processo}

Descrição do processo de implementação dos programas: características, configurações e treinamento das equipes, monitoração e avaliação (Essock e Kontos, 1995; Mowbray et al., 1997b).

Mowbray et al. (1997b) documentaram o processo de disseminação e implementação de novas equipes PACT. A disseminação foi favorecida pelos achados positivos de desfechos pós-implementação em uma avaliação da primeira replicação do modelo PACT, o Harbinger of Grand Rapids. A partir de então, novos programas poderiam ser criados, desde que obedecessem aos princípios tradicionais do PACT. O treinamento das equipes era planejado e executado tendo como modelos programas já estabelecidos, como Harbinger e Madison. Os membros da nova equipe deveriam fazer um estágio para treinamento nos programas-modelo e as novas equipes eram monitoradas regularmente por especialistas do Departamento de Saúde Mental de Michigan. Exigia-se a participação em uma avaliação central de desfechos em que seriam comparadas situações anteriores e posteriores à entrada no PACT. Os autores apontaram que, com adequados treinamento, assistência técnica e monitoração, as replicações também seriam responsáveis por resultados positivos tanto nos desfechos dos clientes como na dinâmica do sistema de saúde mental.

\section{Perfil da população-alvo}

Estudo sobre o perfil da população-alvo, visando fornecer elementos que orientem/facilitem a adequação dos programas às especificidades de cada grupo de pacientes (Goering et al., 1992; Rose et al., 1991).

Rose et al. (1991) avaliaram um programa de case management intensivo para pacientes com alto uso de serviços de emergência, longa permanência em hospitais psiquiátricos e desabrigados com doença mental crônica visando investigar, dentre os primeiros pacientes referidos ao serviço, a presença de história de abuso físico e/ou sexual não detectada anteriormente. Foram encontradas histórias de abuso sexual na infância em 34\% dos pacientes entrevistados, $41 \%$ das mulheres e $9 \%$ dos homens. Entre os pacientes que sofreram abuso, 70\% tinham pais alcoólatras. Os autores concluíram que a investigação de rotina sobre história pregressa de abuso físico ou sexual e o desenvolvimento de ações de suporte para as vítimas devem receber especial consideração no planejamento das políticas de saúde mental, uma vez que a "interação contínua entre abuso indetectado, ser filho de pais alcoólatras, e sistemas pouco responsivos alimenta números crescentes de clientes [...] que estão em alto risco/alto uso de serviços psiquiátricos de internação para pacientes agudos e emergências" (Rose et al., 1991, p. 502).

\section{Equipe terapêutica}

Estudo sobre características da equipe terapêutica (Kirk et al. 1993).

Kirk et al. (1993), em um estudo de coorte, avaliaram mudanças na saúde, na vida pessoal e nas atitudes dos terapeutas de case management intensivo um ano e meio após sua entrada no serviço. Os dados foram coletados na ocasião da contratação e em três tempos posteriores. Detectaram-se aumento do estresse no trabalho, sensação de exaustão emocional e sintomas físicos/depressivos relacionados ao estresse. Os profissionais tenderam a despersonalizar cada vez mais os clientes. No entanto, não houve mudanças na percepção sobre rendimento e na satisfação com o trabalho. Diante de tais resultados, os autores mencionam a importância de iniciativas de suporte para dificuldades no trabalho.

A sétima categoria refere-se ao estudo de satisfação com o PACT e o case management. O objetivo dos estudos é acessar o grau de satisfação com os programas a partir de avaliação de indicadores determinados pelos pesquisadores, coleta de dados sobre satisfação com características ou práticas específicas do programa ou aplicação de instrumentos de avaliação que utilizam perguntas abertas (Dixon et al., 1999; Gerber e Prince, 1999; Huxley e Warner, 1992; McGrew et al., 2002).

Gerber e Prince (1999) avaliaram a satisfação de clientes de PACT a partir de respostas de questionários enviados pelo correio. A maioria dos clientes apresentou opiniões positivas em relação às características dos programas, mostrando-se satisfeitos com o plano terapêutico, a cooperação entre os membros da equipe e os cuidados recebidos. Para a maior parte dos entrevistados, os membros da equipe eram confiáveis, os terapeutas de referência eram capazes de entender os problemas dos pacientes e o tempo que os clientes passavam com seus terapeutas era satisfatório e de grande ajuda. Ademais, a maioria dos clientes considerou que suas necessidades eram adequadamente contempladas e que foram mais ajudados no PACT do que em outros serviços comunitários de tratamento. Dentre os aspectos percebidos como não satisfatórios estavam efeitos colaterais e uso de grandes quantidades das medicações, o grau de influência do paciente sobre seu plano terapêutico, orientação 
sobre opções terapêuticas, informações sobre acesso a seus prontuários e o tipo e a quantidade de cuidado recebido.

Finalmente, a oitava categoria refere-se a revisões bibliográficas ou metanálises sobre PACT e case management. Os estudos podem ter como objetivos descrever os programas e suas replicações ou adaptações, delinear o estado da pesquisa sobre o tema, apontar direções para futuras investigações, fazer comparações com outros serviços (Tabela 2) e análises de desfechos ou de eficácia, demonstrar benefícios para as populações-alvo e fazer comparações entre o PACT e o case management (Bandeira et al., 1998; Burns e Santos, 1995; Drake; Burns, 1995; Lang et al., 1999; Olfson, 1990; Phillips et al., 2001; Taube et al., 1990; Ziguras e Stuart, 2000).

Taube et al. (1990), em revisão bibliográfica sobre PACT, descrevem o programa, o comparam com abordagens tradicionais de cuidado comunitário - em especial programas dos centros comunitários de saúde mental (Tabela 2) - e sistematizam algumas direções para o futuro da pesquisa sobre o tema. Os autores afirmam que "os estudos futuros devem complementar a pesquisa anterior por meio da comparação dos programas com tratamentos atuais e ideais nos centros comunitários de saúde mental ou programas de saúde mental do município; [...] análise dos desfechos dos clientes em programas já estabelecidos por períodos maiores; padronizar as medidas dos vários desfechos dos clientes; e determinar impacto de elementos individuais do programa - isoladamente e associados - em diferentes subgrupos de clientes" (Taube et al., 1990, p. 642).

Burns e Santos (1995) compilaram estudos clínicos randomizados sobre o PACT para pacientes com doença mental grave para realizar uma revisão da pesquisa sobre o assunto e também apontar direções para futuros estudos. Os autores afirmaram que o PACT era a única abordagem terapêutica avaliada por vários estudos clínicos randomizados e que "a pesquisa [...] avançou [...] muito por meio da provisão de evidências dos efeitos positivos [...] na redução do uso hospitalar e no aumento da satisfação do paciente e da família; pela avaliação [...] em um grande espectro de populações, incluindo pacientes com esquizofrenia de início recente, veteranos, clientes com diagnóstico duplo, e desabrigados; e por meio do estudo da integração de outros métodos validados empiricamente como psicoeducação familiar no tratamento assertivo na comunidade" (idem, p. 674). Os autores também apontaram a necessidade de estudos sobre aspectos qualitativos e variações da efetividade do PACT quando aplicado em diferentes tipos de população.

Ziguras e Stuart (2000) também abordaram o tema "PACT versus case management" em sua metanálise. Os autores compararam estudos controlados sobre case management com estudos sobre outros serviços de saúde mental visando abordar a efetividade do case management e comparar as medidas de desfecho entre PACT e case manage- ment clínico. Evidenciou-se maior efetividade do case management - em relação a outros serviços de saúde mental - em diminuir a sensação de sobrecarga familiar, aumentar a satisfação da família e do paciente com os serviços, reduzir os sintomas e as taxas de abandono de tratamento, aumentar o contato do paciente com os serviços de saúde, melhorar o funcionamento social e diminuir o número total de dias de internação. No entanto, quando comparado ao PACT, viu-se que este lhe fora superior nos quesitos melhora do funcionamento social e diminuição do número total de dias de internação.

\section{DISCUSSÃO}

A partir desta revisão, podemos observar que a produção científica sobre o tema é bastante rica e nos permite compreender e discutir os conceitos de PACT e case management e também acessar seus efeitos sobre o manejo terapêutico e a vida de portadores de doença mental grave e persistente.

Tanto o PACT quanto o case management são modelos conhecidos pela sua flexibilidade, característica importante dada a heterogeneidade de suas populações-alvo. Ademais, "as questões em relação ao oferecimento de cuidados comunitários apropriados estão constantemente evoluindo e sendo redefinidas. Para obter sucesso a longo prazo, um modelo de assistência deve acompanhar ativamente os processos de mudança [...]" (Thompson et al., 1990, p. 631). A flexibilidade pode propiciar um maior alcance da abordagem, proporcionando maior acessibilidade do cuidado.

Exemplos da flexibilidade destes modelos são a adaptabilidade dos programas e a capacidade de se associar a outras modalidades de cuidado, oferecendo desfechos ainda melhores, especialmente para pacientes com alto nível de disfuncionalidade (Clark e Rich, 2003; Macias et al., 1994).

Uma questão importante sobre as adaptações do PACT e do case management é o risco de "diluição" da essência dos programas em meio a tantas mudanças em relação ao modelo original (Essock e Kontos, 1995), comprometendo a eficácia do tratamento. Torna-se clara, então, a necessidade de identificar os elementos essenciais dos programas, ou seja, aqueles responsáveis pela reconhecida capacidade do PACT e do case management de oferecer melhores desfechos (McGrew et al., 2003; Schaedle et al., 2002). Uma vez identificados, a fidelidade a eles é questão importante nos processos de adaptação e replicação, havendo, inclusive, uma escala de fidelidade, a dartmouth assertive community treatment fidelity scale ou DACTS (Phillips et al., 2001). Phillips et al. (2001) apontam os elementos indicadores de fidelidade do PACT (Quadro 1). Programas com maior fidelidade alcançariam melhores rendimentos terapêuticos (McHugo et al., 1999). 
Quadro 1 . Elementos indicadores de alta fidelidade no PACT

Presença de baixa taxa cliente-terapeuta.

Compartilhamento dos casos, preconizando 0 atendimento em equipe.

Reuniões de equipe freqüentes e regulares para planejar/rever o projeto terapêutico de cada paciente.

Contatos freqüentes com o cliente e acompanhamento intensivo e oferecido onde 0 paciente está (in vivo).

Desenvolvimento de habilidades para a vida em comunidade.

Continuidade dos cuidadores; acompanhamento contínuo e prolongado, com abordagem assertiva.

Disponibilidade da equipe de 24 horas.

Presença na equipe de psiquiatra, enfermeiro, especialistas em orientação vocacional e em uso de álcool/substâncias.

Supervisor de equipe que também ofereça diretamente serviços aos pacientes.

Presença de pelo menos 10 profissionais que trabalhem em tempo integral, garantindo suficiente diversidade da equipe.

Critérios de admissão de clientes bem definidos, com baixa taxa de entrada e população-alvo bem definida.

Cuidados terapêuticos e outros serviços (por exemplo, suporte habitacional/laborativo, ações de reabilitação) oferecidos diretamente pela equipe.

Disponibilidade para manejar situações de crise, acompanhar a internação hospitalar e planejar a alta.

Abordagens específicas para usuários de álcool e drogas ou pacientes com diagnóstico duplo (incluindo realização de grupos terapêuticos).

Não fechamento de casos (manter algum ponto de contato com todos os clientes).

Apoio/orientação para os integrantes da rede de suporte dos pacientes (familiares, vizinhos, empregadores etc.).

Apoio/orientação e participação de pacientes na equipe terapêutica.

Adaptação da tabela de Phillips et al., 2001, p. 774.

O sucesso terapêutico do PACT e do case management é produto de suas práticas inovadoras. As ações de treinamento em habilidades diárias, estímulo à independência na vida financeira, apoio no manejo da medicação e suporte em questões sociais/laborativas e de moradia são caminhos para se alcançar maior autonomia, facilitar a adaptação à vida em comunidade, aumentar a responsabilidade do paciente pelo seu próprio tratamento e reforçar a autoestima. As iniciativas de suporte/orientação para familiares contribuem na diminuição da sensação de sobrecarga. As ações de agenciamento estimulam e aumentam o uso dos serviços/recursos comunitários. O atendimento realizado na comunidade permite abordar as questões no momento e local onde ocorrem, "melhorando o acesso dos terapeutas aos problemas dos seus pacientes [...]. Isso parece aumentar o impacto terapêutico das intervenções clínicas" (Thompson et al., 1990, p. 630-631). A total disponibilidade da equipe e o acompanhamento regular e assertivo são essenciais na continuidade do cuidado para pacientes "instáveis e difíceis de tratar na comunidade" (Bush et al., 1990, p. 649). A multidisciplinaridade oferece um olhar diversificado tanto do adoecimento quanto do cuidado, possibilitando o oferecimento de uma assistência mais completa e complexa. Ademais, a baixa taxa cliente-terapeuta e o compartilha- mento de casos podem prevenir a exaustão dos membros da equipe (Bachrach, 1992).

Os efeitos destas práticas sobre os desfechos dos pacientes têm sido sistematicamente avaliados e o que se observa freqüentemente é que o PACT, o case management e suas adaptações são mais eficazes do que as iniciativas tradicionais de assistência em melhorar as atitudes em relação a portadores de transtornos mentais, diminuir o uso de álcool/drogas nos pacientes com duplo diagnóstico, melhorar e estabilizar as situações laborativa e habitacional, diminuir as recidivas em uma população de alto risco, diminuir os sintomas, diminuir o uso de serviços de emergência, aumentar a satisfação com o tratamento, melhorar a qualidade de vida e o rendimento nas atividades de vida diária, aumentar a autonomia, impulsionar o apoio familiar e diminuir a sensação de sobrecarga, aumentar a adesão ao tratamento, estabilizar a vida na comunidade, melhorar o funcionamento familiar/laborativo/social, aumentar a rede social e diminuir o isolamento, reduzir o risco de violência e aumentar o uso dos serviços/recursos comunitários entre a população de desabrigados portadores de doença mental (Becker et al., 1999; Borland et al., 1999; Bush et al., 1990; Clark e Rich, 2003; Dixon et al., 1993-a; Dixon et al., 1993-b; Dixon et al., 1997; Durell et al., 1993; Dvoskin e Steadman, 1994; Gerber e Prince, 1999; Goering et al., 1988; Herinckix et al., 1997; Lafave et al., 1996; Macias et al., 1994; McGrew et al., 1995; McHugo et al., 1999; Morse et al., 1992; Neale e Rosenheck, 1995; No authors listed, 2000; Sherman e Porter, 1991; Wasylenki et al., 1993; Yang et al., 2005).

A superioridade do PACT e do case management em diminuir o número e a duração das internações psiquiátricas está entre os desfechos mais importantes e aparece repetidamente nos estudos de avaliação e comparação dos modelos (Bond et al., 1988; Dincin et al., 1993; Lafave et al., 1996; Macias et al., 1994; McGrew et al., 1995; McHugo et al., 1999; Santos et al., 1993; Tibbo et al., 1999; Yang et al., 2005). Alguns estudos constataram que o aumento da intensidade dos cuidados de case management não foi mais eficaz em reduzir a freqüência das internações (Curtis et al., 1992; Hornstra et al., 1993) e que, comparado aos serviços usuais, o case management aumentou o uso hospitalar (Franklin et al., 1987). No entanto, Olfson (1990), em revisão de estudos experimentais sobre PACT, classificou a diminuição do uso hospitalar como "o achado mais consistente" (idem, p. 640).

As diferenças entre o PACT e o case management também são objetos de análises e "revisões da pesquisa concluem sistematicamente que, comparado com outros tratamentos sob condições controladas, o tratamento assertivo na comunidade resulta maior redução da hospitalização psiquiátrica [...]" (Phillips et al., 2001, p. 771). Além disso, Ziguras e Stuart (2000) apontaram a superioridade do PACT em relação ao case management nos quesitos melhora do 
funcionamento social e diminuição do número total de dias de internação.

É importante apontar para uma discussão sobre a possibilidade de as práticas terapêuticas terem como real conseqüência o reforço das situações de dependência, uma vez que tais iniciativas "constituem uma matriz de serviços paternalistas e, por vezes, coercitivos [...]" (Thompson et al., 1990, p. 631). Segundo os autores, práticas como "reabilitação vocacional; [...] aumento do uso de grupos, especialmente os de auto-ajuda; o desenvolvimento de [...] serviços operados por clientes; o uso da mídia e psicoeducação para aumentar a aceitação do doente mental [...]; e o reforço do direito dos pacientes de reclamar ou até mesmo recusar serviços [...]" (ibidem, p. 632) poderiam diminuir este suposto caráter coercitivo e perpetuador de dependência e segregação de maneira apenas limitada. Em confronto a este questionamento, temos que o desfecho "melhora/aumento da vida independente" é reproduzido sistematicamente em estudos de avaliação (Goering et al., 1988; Lafave et al., 1996; McGrew et al., 1995).

\section{CONCLUSÃO}

Podemos concluir que o PACT e o case management são iniciativas importantes e que, quando comparados a outros sistemas de cuidado em saúde mental, são modelos reconhecidamente mais eficazes em trazer evoluções mais favoráveis para portadores de doença mental grave e persistente. Tal conclusão pode ser verificada pelos achados de resultados fortemente positivos em relação a desfechos, satisfação com o tratamento e eficácia global dos modelos.

A flexibilidade e a adaptabilidade dos modelos, bem como a possibilidade da associação, com sucesso, a outras iniciativas terapêuticas (Clark e Rich, 2003), favorecem o oferecimento de maior acessibilidade do cuidado, especialmente diante da heterogeneidade das populações-alvo. Além da maior acessibilidade, a abordagem multidisciplinar e as ações terapêuticas orientadas não só para controlar os sintomas, como também para reinserir/adaptar à vida em comunidade, diminuir as internações, aumentar a autonomia e melhorar o funcionamento familiar/social/laborativo são ferramentas importantes para acessar a complexidade do adoecimento psíquico grave e podem ser consideradas catalisadoras do sucesso terapêutico dos modelos.

\section{REFERÊNCIAS}

Amarante P. 0 homem e a serpente - outras histórias para a loucura e a psiquiatria, 1a reimpressão da 1 a ed, Rio de Janeiro, Ed. Fiocruz, 2000

Bachrach LL. Case management revisited. Hosp Community Psychiatry, 43(3): 209-210, 1992.
Bachrach LL. Case management: toward a shared definition. Hosp Community Psychiatry, 40(9): 883-884, 1989.

Bachrach LL. Continuity of care and approaches to case management for long-term mentally ill patients. Hosp Community Psychiatry, 44(5): 465-468, 1993.

Bandeira M, Gelinas D, Lesage A. Desinstitucionalização: o programa de acompanhamento intensivo na comunidade". J Bras Psiquitr 47(12): 627-640, 1998.

Becker RE, Meisler N, Stormer G,. Brondino MJ. Employment outcomes for clients with severe mental illness in a PACT model replication. Psychiatric Services, 50(1): 104-106, 1999.

Bond GR, Miller LD, Krumwied RD, Ward RS. Assertive case management in three CMHCs: a controlled study. Hosp Community Psychiatry, 39: 411-418, 1988.

Borland A, McRae J, Lycan C. Outcomes of five years of continuous intensive case management. Hospital and Community Psychiatry, 40:369-376, 1989.

Burns BJ, Santos AB. Assertive community treatment: an update of randomized trials. Psychiatric Services, 46(7): 669-675, 1995

Bush CT, Langford MW, Rosen P, Gott W. Operation outreach: intensive case management for severely psychiatrically disabled adults. Hosp Community Psychiatry, 41(6): 647-649, 1990

Cavalcanti, MT. 0 tear das cinzas: um estudo sobre as relações entre psicose e instituição psiquiátrica. - Rio de Janeiro, UFRJ, IPUB, 1992

Clark C, Rich AR. Outcomes of Homeless Adults With Mental IIIness in a Housing Program and in Case Management Only. Psychiatric Services 54(1): 78-83, 2003

Curtis JL, Millman EJ, Struening E, D'Ercole A. Deaths among former psychiatric inpatients in an outreach case management program. Psychiatric Services, 47(4): 398-402, 1996.

Curtis JL, Millman EJ, Struening E, D'Ercole A. effect of case management on rehospitalization and utilization of ambulatory care services. Hosp Community Psychiatry, 43(9): 895-899, 1992.

Deci PA, Santos AB, Hiott DW, Schoenwald S, Dias JK. Dissemination of assertive community treatment programs. Psychiatric Services, 46(7): 676-678, 1995

Dietzen $L L$, Bond GR. Relationship between case manager contact and outcome for frequently hospitalized psychiatric clients. Hosp Community Psychiatry, 44: 839-843, 1993.

Dincin J, Wasmer D, Witheridge TF, Sobeck L, Cook J, Razzano L. Impact of assertive community treatment on the use of state hospital inpatient bed-days. Hosp Community Psychiatry, 44:833-838, 1993.

Dixon L. Assertive Community Treatment: twenty-five years of gold. Psychiatric Services, 51(6): 759-765, 2000(a).

Dixon L, Friedman N, Lehman A. Compliance of homeless mentally ill persons with assertive community treatment. Hosp Community Psychiatry, 44(6):581-583, 1993(b).

Dixon L, Friedman N, Lehman A. Housing patterns of homeless mentally ill persons receiving assertive treatment services. Hosp Community Psychiatry, 44(3): 286-288, 1993(a).

Dixon L, Hackman A, Lehman A. Consumers as staff in assertive community treatment programs. Adm Policy Ment Health, 25(2): 199-208, 1997.

Dixon L, Lucksted A, Stewart B, Delahanty J. Therapists' contacts with family members of persons with severe mental illness in a community treatment program. Psychiatric Services, 51(11): 1449-1451, 2000(b).

Dixon L, Turner J, Krauss N, Scott J, McNary S. Case managers' and clients' perspectives on a representative payee program. Psychiatric Services, 50: 781-786, 1999.

Drake RE, Burns BJ. Special section on assertive community treatment: an introduction. Psychiatric Services, 46(7): 667-668, 1995.

Durell J, Lechtenberg B, Corse S, Frances RJ. Intensive case management of persons with chronic mental illness who abuse substances. Hosp Community Psychiatry, 44(5): 415-428, 1993.

Dvoskin JA, Steadman HJ. Using intensive case management to reduce violence by mentally ill persons in the community. Hosp Community Psychiatry, 45: 679-684, 1994.

Essock SM, Kontos N. Implementing assertive community treatment teams. Psychiatric Services, 46(7): 679-683, 1995

Fariello D, Scheidt, S. Clinical case management of the dually diagnosed patient. Hosp Community Psychiatry, 40(10):1065-1067, 1989.

Fiander M, Burns T. A Delphi approach to describing service models of community mental health practice. Psychiatric Services, 51(5):.656-658, 2000 
Franklin JL, Solovitz B, Mason M, Clemons JR, Miller GE. An evaluation of case management. Am J Public Health, 77(6): 674-678, 1987.

Gerber GJ, Prince PN. Measuring client satisfaction with assertive community treatment. Psychiatric Services, 50(4): 546-550, 1999.

Goering P, Wasylenki D, Onge M, Paduchak D, Lancee W. Gender differences among clients of a case management program for the homeless. Hosp Community Psychiatry, 43(2): 160-165, 1992.

Goering PN, Wasylenki DA, Lancee WJ, Farkas M, Ballantyne R. What difference does case management make?. Hosp Community Psychiatry, 39: 272-276, 1988.

Herinckx HA, Kinney RF, Clarke GN, Paulson RI. Assertive community treatment versus usual care in engaging and retaining clients with severe mental illness. Psychiatric Services, 48(10):1297-1306, 1997.

Hornstra RK, Wolfe VB, Sagduyu K, Riffle DW. The effect of intensive case managenent on hospitalization of patients with schizophrenia. Hosp Community Psychiatry, 44(9): 844-847, 1993.

Huxley P, Warner R. Case management, quality of life, and satisfaction with services of longterm psychiatric patients. Hosp Community Psychiatry, 43(8):799-802, 1992.

Joannette JA, Lawson JS, Eastabrook SJ, Krupa T. Community tenure of people with serious mental illness in assertive community treatment in Canada. Psychiatric Services, 56(11): 1387-1393, 2005.

Kanter J. Clinical case management: definition, principles, components. Hosp Community Psychiatry, 40: 361-368, 1989.

Kanter J, Silva M. Case management and managed care: investing in recovery. Psychiatric Services 47(7): 699-701, 1996

King R, Bas JL, Spooner D. The impact of caseload on the personal efficacy of mental health case managers. Psychiatric Services, 51(3): 364-368, 2000

Kirk SA, Koeske GF, Koeske RD. Changes in health and job attitudes of case managers providing intensive services. Hosp Community Psychiatry, 44: 168-173, 1993.

Lafave HG, Souza HR, Gerber GJ. Assertive community treatment of severe mental illness: a canadian experience. Psychiatric Services, 47(7): 757-759, 1996.

Lang MA, Davidson L, Bailey P, Levine MS. Clinicians' and clients' perspectives on the impact of assertive community treatment. Psychiatric Services, 50(10): 1331-1340, 1999.

Liberman RP, Kopelowicz A. Rehab rounds: teaching persons with severe mental disabilities to be their own case managers. Psychiatric Services, 53(11): 1377-1379, 2002.

Macias C, Kinney R, Farley OW, Jackson R, Vos B. The role of case management within a community support system: partnership with psychosocial rehabilitation. Community Ment Health J, 30(4): 323-339, 1994.

McGrew JH, Bond GR, Dietzen L, McKasson M, Miller LD. A multisite study of client outcomes in assertive community treatment. Psychiatric Services, 46(7): 696-701, 1995.

McGrew JH, Pescosolido B, Wright E. Case managers' perspectives on critical ingredients of assertive community treatment and on its implementation. Psychiatric Services, 54(3): $370-376,2003$

McGrew JH, Wilson RG, Bond GR. An exploratory study of what clients like least about assertive community treatment. Psychiatric Services, 53(6): 761-763, 2002.

McHugo GJ, Drake RE, Teague GB, Xie H. Fidelity to assertive community treatment and client outcomes in the New Hampshire dual disorders study. Psychiatric Services, 50(6): 818-824, 1999.

McRae J, Higgins M, Lycan C, Sherman W. What happens to patients after five tears of intensive case management stops?. Hosp Community Psychiatry, 41: 175-179, 1990.

Meisler N, Santos AB, Rowland MD, Smith S, Molloy M, Tyson S. Bridging the gap between inpatient and outpatient providers using organizational elements of assertive community treatment. Adm Policy Ment Health, 25(2): 141-152, 1997.

Morse GA, Calsyn RJ, Allen G, Tempethoff B, Smith R. Experimental comparision of the effects of three treatment programs for homeless mentally ill people. Hosp Community Psychiatry, 43: 1005-1010, 1992.
Mowbray CT, Collins MR, Plum TB, Masterton T, Mulder R. Harbinger I: The development and evaluation of the first PACT replication. Adm Policy Ment Health, 25(2): 105-123, 1997 (a).

Mowbray CT, Plum TB, Masterton T. Harbinger II: development and evolution of assertive community treatment in Michigan. Adm Policy Ment Health, 25(2):125-139, 1997(b).

Neale MS, Rosenheck RA. Therapeutic alliance and outcome in a VA intensive case management program. Psychiatric Services, 46(7): 719-721, 1995.

Neale MS, Rosenheck RA. Therapeutic limit setting in an assertive community treatment program. Psychiatric Services, 51(4): 499-505, 2000.

Nikkel RE, Smith G, Edwards D. A Consumer-Operated Case Management Project. Hosp Community Psychiatry 43(6): 577-579, 1992.

No authors listed. Gold award: a community treatment program--Mendota Mental Health Institute Madison, Wisconsin. 1974. Psychiatric Services, 51(6): 755-758, 2000.

Olfson M. Assertive community treatment: an evaluation of the experimental evidence. Hosp Community Psychiatry, 41(6): 634-641, 1990.

Phillips SD, Burns BJ, Edgar ER, Mueser KT, Linkins KW, Rosenheck RA, Drake RE, Herr EM. Moving assertive community treatment into standard practice. Psychiatric Services, 52(6): 771-779, 2001.

Ridgely MS, Morrissey JP, Paulson RI, Goldman HH, Calloway MO. Characteristics on activities of case managers in the rwj foundation program on chronic mental illness. Psychiatric Services, 47(7): 737-743, 1996.

Rose SM, Peabody CG, Stratigeas B. Undetected abuse among intensive case management clients. Hosp Community Psychiatry, 42(5): 499-503, 1991.

Rosenheck RA, Neale MS. Therapeutic limit setting and six-month outcomes in a veterans affairs assertive community treatment program. Psychiatric Services, 55(2): 139-144, 2004.

Santos AB, Deci PA, Lachance KR, Dias JK, Sloop TB, Hiers TG, Bevilacqua JJ. Providing assertive community treatment fo severely mentally ill patients in a rural area. Hosp Community Psychiatry, 44: 34-39, 1993.

Schaedle R, McGrew JH, Bond GR, Epstein I. A comparision of experts' perspectives on assertive community treatment and intensive case management. Psychiatric Services, 53(2): 207210, 2002.

Shaw RE, Hargreaves WA, Surber R, Luft L, Shadoan R. Continuity and Intensity of case management activity in three CMHCs. Hosp Community Psychiatry, 41(3): 323-326, 1990.

Sherman 0S, Porter R. Mental health comsumers as case management aides. Hosp Community Psychiatry, 42(5): 494-498, 1991.

Simon PM, Osofsky HJ, Morse EV, Speier T. Training Older Adults to Work as Psychiatric Case Management Aides. Hosp Community Psychiatry, 44(12): 1162-1165, 1993.

Taube CA, Morlock L, Burns BJ, Santos AB. New Directions in Research on Assertive Community Treatment. Hosp Community Psychiatry 41(6): 642-647, 1990.

Thompson KS, Griffith E, Leaf PJ. A Historical Review of the Madison Model of Community Care. Hosp Community Psychiatry, 41(6): 625-634, 1990.

Tibbo P, Chue P, Wright E. Hospital outcome measures following assertive community treatment in Edmonton, Alberta. Can J Psychiatry, 44: 276-279, 1999.

Wasylenki DA, Goering PN, Lancee W, Lemire D, Lindsey S. The hostel outreach program: assertive case management for homeless mentally ill persons. Hosp Community Psychiatry, 44: 848-853, 1993

Whiteridge, TF. The assertive community treatment worker: an emerging role and its implications for professional training. Hosp Community Psychiatry, 40: 620-624, 1989.

Yang J, Law S, Chow W, Andermann L, Steinberg R, Sadavoy J. Assertive community treatment for persons with severe and persistent mental illness in ethnic minority groups. Psychiatric Services, 56(9): 1053-1055, 2005

Ziguras SJ, Stuart GW. A Meta-analysis of the effectiveness of mental health case management over 20 years. Psychiatric Services, 51(11): 1410-1421, 2000. 